\title{
Pace-and-Drive of the Human Sinoatrial Node - A Preliminary Computational Investigation
}

\author{
Alan Fabbri ${ }^{1}$, Axel Loewe ${ }^{2}$, Ronald Wilders ${ }^{3}$, Stefano Severi ${ }^{1}$ \\ ${ }^{1}$ University of Bologna, Cesena, Italy \\ ${ }^{2}$ Karlsruhe Institute of Technology (KIT), Karlsruhe, Germany \\ ${ }^{3}$ Academic Medical Center (AMC), Amsterdam, The Netherlands
}

\begin{abstract}
The sinoatrial node (SAN) is the natural pacemaker of our heart. How this small tissue is able to drive a remarkably larger number of intrinsically quiescent atrial cells is still debated; a computational investigation of the underlying mechanisms can help to better understand the SAN's ability to pace-and-drive the surrounding atrium.

Aim of this work is to elucidate how the human SAN action potential can successfully be captured by and propagate into the surrounding atrial tissue.

The Fabbri et al. and the Courtemanche et al. models were used to describe the human SAN and atrial cells, respectively. The behaviour of two coupled regions was investigated varying the interregional conductivity ( $\sigma$ ) and relative size. Simulations showed that it requires at least an isopotential SAN region 2.85 times wider than the atrial one. A $1 D$ strand of homogeneously coupled SAN and atrial elements was used to identify an interval for $\sigma$ showing pace-and-drive behaviour (100 SAN vs 100 atrial elements) and to investigate the source-sink interplay (10, 50 or 100 SAN elements vs 100 atrial elements). The $1 D$ strand showed pace-and-drive behaviour for $\sigma=[0.08-36] \mathrm{S} / \mathrm{m}$; a stronger source, with a higher number of SAN elements, led to a wider $\sigma$ range that allowed pace-and-drive behaviour, whereas a stronger sink did not affect the behaviour of the tissue.

This preliminary work shows the ability of a small human SAN region to pace-and-drive the surrounding atrial tissue. Further investigations are needed to explore different conductivity configurations, including spatial gradients.
\end{abstract}

\section{Introduction}

The sinoatrial node (SAN) is a small, specialized tissue located in the right atrium and responsible for the physiological heart rate in healthy subjects. Its selfoscillating action potential (AP) starts the chain of events that leads to the electrical activation and the subsequent mechanical contraction of the whole heart. When two or more cardiac cells are coupled, the behaviour of each cell is not only determined by its intrinsic properties governing membrane ion currents but also by the electrotonic currents that flow through the gap junctions shared with neighbouring cells. Experimental work by Jalife [1] showed that two pacemaker centres beating at different frequencies mutually entrained and reached a common cycle length (CL) intermediate between the mean period of the fast and the slow one, once electrically coupled. A computational study by Joyner et al. [2] showed that regional changes in membrane properties (e.g. plateau currents) affected the spatial distribution of the AP properties along the tissue due to the effects of electrotonic currents.

The propagation of the electrical signal from the SAN tissue to the atrial tissue was investigated in animal models, especially in rabbits, starting from the 1980s. The fair amount of experimental data provided a strong base for building computational models, which investigated the required level of coupling to successfully pace-anddrive the atrial tissue. Among them, Joyner and van Capelle [3] systematically explored the behaviour of two coupled regions and of 2D radially symmetric cardiac tissue slices composed of sinoatrial and atrial rabbitspecific cell models.

Recent studies by Chandler et al. [4] and Csepe et al. [5] shed light on the anatomical structure and conduction pathways of the human SAN. However, a computational model able to describe the propagation of the electrical stimulus from the SAN to the atrial human tissue is still lacking.

The aims of the present work are 1) characterizing the behaviour of two discrete electrically coupled SAN and atrial regions when varying the membrane surface and the gap junction conductivity, 2) identifying the range of conductivity that yields a pace-and-drive behaviour in a homogeneous 1D strand, and 3) investigating the sourcesink interplay by varying the number of SAN and atrial elements in the strand. 


\section{Methods}

In order to investigate the mechanisms involved in the electrical propagation of the AP in human sinoatrial and atrial tissue, the human-specific cell models by Fabbri et al. [6] and Courtemanche et al. [7], for the sinoatrial node and for the atrial cells, respectively, were chosen.

\section{Coupling of discrete sinoatrial node and atrial regions}

The study about the electrical interaction between two discrete regions (SAN and atrial) was performed varying two parameters that describe the strength of the coupling, i.e. the conductivity $(\sigma[\mathrm{S}])$ and the dimensions of the investigated region $\left(\mathrm{k}_{\text {ratio }}[-]\right)$.

In particular, $\mathrm{k}_{\text {ratio }}$ is defined as:

$$
k_{\text {ratio }}=\frac{C_{S A N, \text { cell }}}{C_{A T R, \text { cell }}} \frac{S_{A T R}}{S_{S A N}}
$$

where $C_{S A N, \text { cell }}=57 \mathrm{pF}[6]$ and $C_{A T R, \text { cell }}=100 \mathrm{pF}$ [7]. The terms relative to the membrane surface, $\mathrm{S}_{\mathrm{SAN}}$ and $\mathrm{S}_{\mathrm{ATR}}$, affect the calculation of the coupling current among the two tissue regions. In this sense, they represent the amount of membrane of one region which is involved in the coupling with the other.

The behaviour of the coupled-regions system was tested for $\sigma$ between $10^{-8}$ and $5 \cdot 10^{-5} \mathrm{~S}$ and $k_{\text {ratio }}$ between $0.057\left(\mathrm{~S}_{\mathrm{SAN}}\right.$ ten times bigger than $\left.\mathrm{S}_{\mathrm{ATR}}\right)$ and 0.57 $\left(\mathrm{S}_{\mathrm{SAN}}=\mathrm{S}_{\mathrm{ATR}}\right)$.

\section{$1 D$ tissue strand}

The monodomain model was adopted to describe the propagation of the electrical stimulus from the sinoatrial to the atrial tissue. The identification of a range of conductivity values that yields pace-and-drive behaviour was obtained through a mono-dimensional strand composed of 100 sinoatrial $(1 \mathrm{~cm})$ and 100 atrial cellular elements $(1 \mathrm{~cm})$; each element had a side length of 100 $\mu \mathrm{m}$. We employed a constant value of conductivity $\sigma_{\mathrm{h}}$ $[\mathrm{S} / \mathrm{m}]$ along the whole tissue, simulating an electrically homogeneous strand.

The source-sink interplay was investigated adopting a number of SAN cells equal to 10,50 or 100 against 100 or 200 atrial cells. The strength of the source and the sink was investigated by comparing the cycle length (CL) of the leftmost sinoatrial element (index \#0) with the CL relative to an uncoupled setup $\left(\sigma_{\mathrm{h}}=0\right)$.

Finally the conduction velocity (CV) was estimated measuring the time delay between the activation of the $25^{\text {th }}$ and $75^{\text {th }}$ atrial cells assuming a constant propagation velocity.

\section{Results}

Coupling of discrete sinoatrial node and atrial regions

The coupled-region system showed three distinct behaviours as can be seen in Figure 1: a) not-pace, where the automaticity of the sinoatrial node is completely lost, b) pace-but-not-drive, where the sinoatrial node keeps the automaticity but it is not able to activate the atrial region, and c) pace-and-drive, where the automatic sinoatrial region APs are successfully captured by the atrial tissue and propagate. $\sigma=9 \cdot 10^{-8} S$ was the maximal value that led to the pace-but-not-drive behaviour irrespective of the value of $k_{\text {ratio }}$. For $k_{\text {ratio }}$ equal to 0.057 and 0.1 the system switched from pace-but-not-drive to pace-anddrive upon increasing $\sigma$, whereas for $k_{\text {ratio }}$ of 0.285 or higher, the not-pace behaviour followed the pace-but-notdrive one.

For $k_{\text {ratio }}=0.1425$ (sinoatrial surface four times higher than the atrial one) the pace-and-drive behaviour was disrupted by a not-pace interval for $\sigma=[3-7] \cdot 10^{-8} \mathrm{~S}$. The outward current provided by the SAN region in the not-pace configuration did not allow the SAN to reach the threshold, stopping in this way the automaticity. For higher values the pace-and-drive behaviour was recovered with a SAN-like AP waveform of the atrial region.

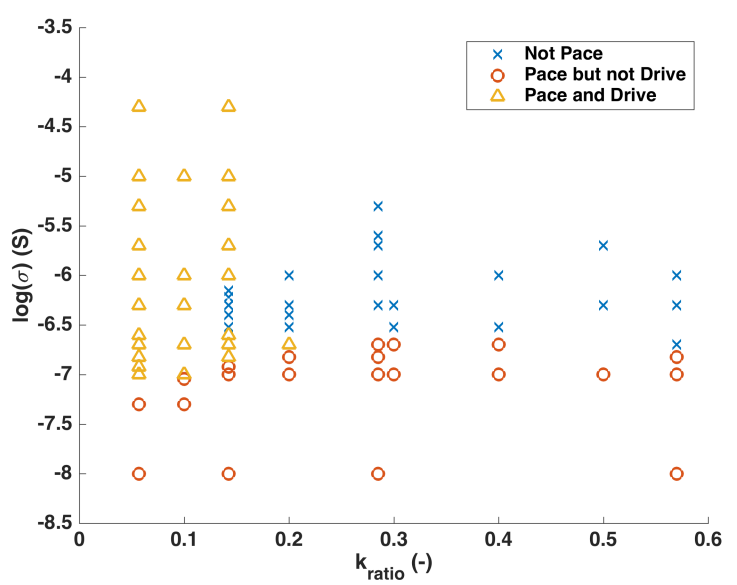

Figure 1: Results of the simulations for two coupled regions: SAN and atrial, varying the membrane surface $\left(\mathrm{k}_{\text {ratio }}\right)$ and the strength of the coupling $(\sigma)$. Not-pace (crosses), pace-but-notdrive (circles) and pace-and-drive (triangles) behaviour was observed.

\section{D tissue strand}

The mono-dimensional homogeneous strand composed of 100 SAN and 100 atrial elements showed a pace-and-drive behaviour for $\sigma_{\mathrm{h}}=[0.08-36.5] \mathrm{S} / \mathrm{m}$. Lower values of $\sigma_{h}$ led to a pace-but-not-drive behaviour, whereas values above stopped the automaticity of the sinoatrial node (not-pace).

Figures 2 and 3 compare the AP waveform and the time course of the fast sodium current $\left(\mathrm{I}_{\mathrm{Na}}\right)$ at the 
transition between SAN and atrial regions for $\sigma_{\mathrm{h}}=1 \mathrm{~S} / \mathrm{m}$. The SAN AP showed a progressive hyperpolarization (MDP $=-66.6$ for the last SAN element versus $-58.9 \mathrm{mV}$ in the uncoupled cell) due to the presence of the atrial tissue (see Fig. 2, panel A). The upstroke of the SAN elements close to the atrium was steeper due to a larger $\mathrm{I}_{\mathrm{Na}}$, almost negligible in cells far from the transitional zone (see Fig. 3, panel B). The atrial elements close to the SAN ones showed a depolarized resting potential in comparison with the single cell model $\left(\mathrm{V}_{\text {rest }} \approx-66 \mathrm{mV}\right.$ and a slight depolarization during the diastolic phase for the first atrial cell, $\mathrm{V}_{\text {rest,uncoupled }} \approx-81 \mathrm{mV}$ ); the typical spikenotch-dome morphology was lost in the first atrial elements, which showed an intermediate waveform between sinus-nodal and atrial. The atrial waveform and the resting potential were recovered after 10 elements (1 $\mathrm{mm}$ ) from the transitional region (Fig. 3, panel A). A more hyperpolarized resting potential led to higher $\mathrm{I}_{\mathrm{Na}}$ availability, remarkably higher than in the first atrial elements (Fig. 3, panel B).
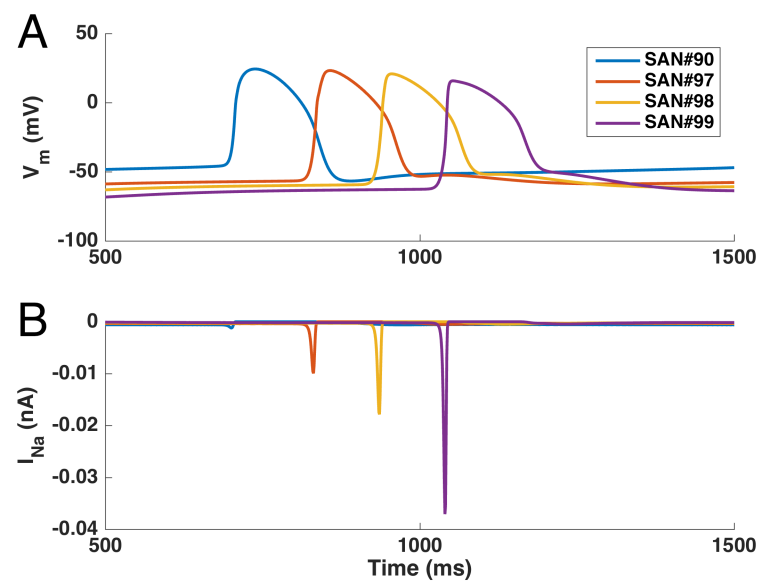

Figure 2: Comparison of A) AP waveform and B) $\mathrm{I}_{\mathrm{Na}}$ time course of SAN elements close to the transitional zone for $\sigma_{\mathrm{h}}=1$ $\mathrm{S} / \mathrm{m} . \mathrm{V}_{\mathrm{m}}$ and $\mathrm{I}_{\mathrm{Na}}$ time courses are delayed by $100 \mathrm{~ms}$ for sake of clarity. Preceding SAN APs look like SAN\#90.
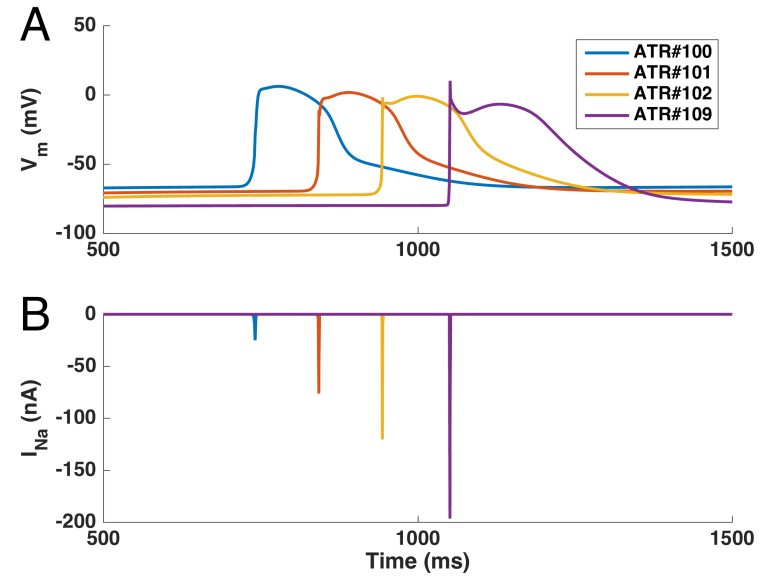

Figure 3: Comparison of A) AP waveform and B) $\mathrm{I}_{\mathrm{Na}}$ time course of atrial elements close to the transitional zone for $\sigma_{\mathrm{h}}=1$
$\mathrm{S} / \mathrm{m} . \mathrm{V}_{\mathrm{m}}$ and $\mathrm{I}_{\mathrm{Na}}$ time courses are delayed by $100 \mathrm{~ms}$ for sake of clarity. Note the different ordinate scale of panel B compared to Figure 2. Following atrial APs look like ATR\#109.

An increase of the number of the sinoatrial elements led to a wider $\sigma_{h}$ range yielding pace-and-drive behaviour. In particular, the upper bound of the interval was shifted towards higher values, with $\sigma_{\mathrm{h}}=0.32,9$ and $36.5 \mathrm{~S} / \mathrm{m}$ for a SAN tissue composed of 10,50 and 100 elements, respectively. Furthermore, a higher number of SAN cells caused a lower CL, i.e. faster beating, at the same value of $\sigma_{h}$ (e.g. for $\sigma_{\mathrm{h}}=0.15 \mathrm{~S} / \mathrm{m}, \mathrm{CL}=963,814$ and $814 \mathrm{~ms}$ for 10,50 and $100 \mathrm{SAN}$ elements). In combination, longer CLs could be obtained using higher numbers of SAN elements $\left(\mathrm{CL}_{\max }=1807,2226\right.$ and 2561 $\mathrm{ms})$ as depicted in the $\sigma_{h}$ vs. CL plot in Figure 4. Changing the number of atrial elements from 100 to 200 did alter behaviour neither qualitatively nor quantitatively.

Estimated CV varied between $0.674 \mathrm{~cm} / \mathrm{s}$ for $\sigma_{\mathrm{h}}=$ $0.08 \mathrm{~S} / \mathrm{m}$ and $\mathrm{CV}=83.3 \mathrm{~cm} / \mathrm{s}$ for $\sigma_{\mathrm{h}}=36.5 \mathrm{~S} / \mathrm{m}$.

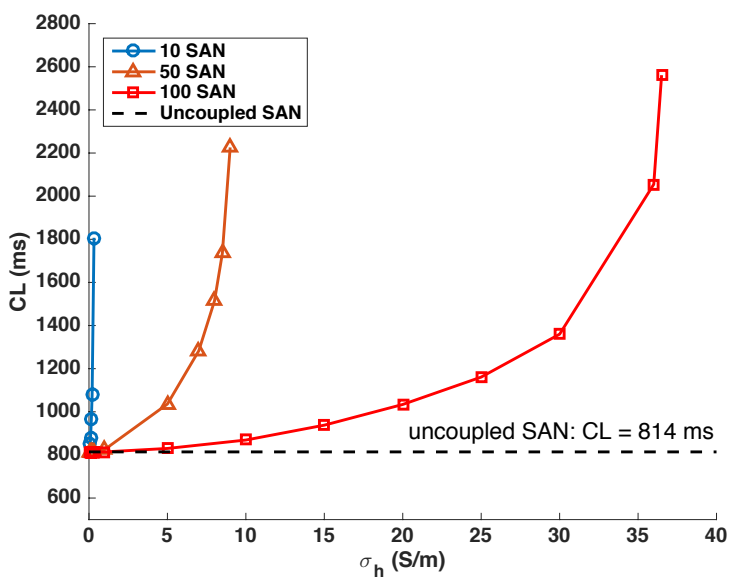

Figure 4: Source-sink interplay. CL of SAN elements at different conductivity values for a homogeneous 1D strand composed of 10,50 or 100 SAN elements against 100 atrial elements. The dashed line indicates the intrinsic CL of the SAN cell elements.

\section{Discussion and Conclusion}

The present work represents a preliminary investigation on the propagation of the AP from the sinoatrial node to the atrial tissue using human-specific computational membrane models.

The in silico experiments showed that the human SAN model is able to pace-and-drive the atrial tissue under specific conditions of electrotonic coupling and cell/tissue dimensions. The system of two discrete coupled regions showed pace-and-drive behaviour for $\mathrm{k}_{\text {ratio }} \leq 0.2$. This value is obtained for a $\mathrm{S}_{\mathrm{SAN}}: \mathrm{S}_{\mathrm{ATR}}$ equal to $2.85: 1$. The assumption of isopotential region is satisfied by two 
coupled cells. In that case the $\mathrm{S}_{\mathrm{SAN}} \mathrm{S}_{\mathrm{ATR}}$ ratio means that 5 SAN cells are needed to successfully excite the atrial one. If we extend the discussion to 2 isopotential tissues, the $\mathrm{S}_{\mathrm{SAN}}: \mathrm{S}_{\mathrm{ATR}}=2.85: 1$ means that the surface of the SAN region that participates to the coupling should be almost three times more extended than the atrial one. This result is in agreement with the study by Joyner \& van Capelle [3], who showed that the isopotential SAN region surface must be wider than the atrial one in order to successfully activate the latter (Figure 3 in [3], panels A and B).

The disruption of the pace-and-drive for $\sigma=[3-7]$. $10^{-8} \mathrm{~S}$ and the following recovery of the driving of the atrial region for higher values of coupling is intriguing. The behaviour showed by our simulations differs from the results obtained by Joyner \& van Capelle [3], where increasing the strength of coupling, keeping the same dimensions of SAN or atrial region, did not show a recovery from not pace. Interestingly, in our simulations, the disruption distinguished two different kinds of paceand-drive, the one with an atrial-like and another with a SAN-like waveform for the atrial region.

The analysis of the behaviour of a 1D strand composed of 100 SAN and 100 atrial elements with homogeneous coupling revealed that the pace-and-drive region in the $\sigma_{h}$ space is quite extended and also that the SAN elements are strong enough to drive the atrial tissue without being electrically shielded from the load generated by the atrium. However the conductivity $\sigma_{h}$ corresponding to a physiological conduction velocity in the atrial tissue $(\mathrm{CV} \approx 85 \mathrm{~cm} / \mathrm{s})$ yields a too long $\mathrm{CL}$ (= $2726 \mathrm{~ms}$ ) compared to physiological heart rates. This discrepancy suggests a non-homogeneous conductivity in vivo.

The SAN elements located in the transitional zone showed a "peripheral-like" AP [8] even if the employed SAN AP model does not distinguish between central and peripheral cells. The enhanced contribution of $\mathrm{I}_{\mathrm{Na}}$ due to the electrical load of the atrial tissue was responsible for the faster upstroke of the SAN elements close to the atrial ones.

The source-sink interplay plays an important role in excitation propagation, especially for the SAN that has to drive the remarkable electrical load generated by the surrounding atrial tissue. Our simulations point out that the range extension of the conductivity that allows the pace-and-drive behaviour highly depends on the number of the SAN elements (strength of the source). The carried out simulations with 100 and 200 atrial elements showed the same behaviour, at the same conditions of source strength. This result suggest that the further downstream 100 atrial elements do not substantially increase the electrical load of the sink.

The focus on simple geometrical setups (two discrete coupled regions and a 1D strand), even if far from a physiological configuration, allowed to observe the fundamental electrical behaviour without confounding factors due to a complex geometry.

Next steps should take into account the cellular and gap junction heterogeneity, introducing differences in ion channel conductances and spatial gradients of coupling $[3,9]$. An appropriate coupling configuration will be also helpful to obtain both a physiological conduction velocity and CL, not achievable in our 1D homogeneous strand.

\section{References}

[1] J. Jalife, "Mutual entrainment and electrical coupling as mechanisms for synchronous firing of rabbit sino-atrial pacemaker cells," J. Physiol., vol. 356, no. 1, pp. 221-243, Nov. 1984.

[2] R. W. Joyner, J. Picone, R. Veenstra, and D. Rawling, "Propagation through electrically coupled cells. Effects of regional changes in membrane properties.," Circ. Res., vol. 53, no. 4, pp. 526-534, Oct. 1983.

[3] R. W. Joyner and F. J. L. van Capelle, "Propagation through electrically coupled cells. How a small SA node drives a large atrium," Biophys. J., vol. 50, no. 6, pp. 1157-1164, Dec. 1986.

[4] N. Chandler et al., "Computer three-dimensional anatomical reconstruction of the human sinus node and a novel paranodal area," Anat. Rec., vol. 294, no. 6, pp. 970-979, Jun. 2011.

[5] T. A. Csepe et al., "Human sinoatrial node structure: 3D microanatomy of sinoatrial conduction pathways," Prog. Biophys. Mol. Biol., vol. 120, no. 1-3, pp. 164-178, Jan. 2016.

[6] A. Fabbri, M. Fantini, R. Wilders, and S. Severi, "Computational analysis of the human sinus node action potential: model development and effects of mutations," $J$. Physiol., vol. 595, no. 7, pp. 2365-2396, Apr. 2017.

[7] M. Courtemanche, R. J. Ramirez, and S. Nattel, "Ionic mechanisms underlying human atrial action potential properties: insights from a mathematical model," Am. J. Physiol. Heart Circ. Physiol., vol. 275, no. 1, pp. H301-H321, Jul. 1998.

[8] N. J. Chandler et al., "Molecular architecture of the human sinus node: insights into the function of the cardiac pacemaker," Circulation, vol. 119, no. 12, pp. 1562-1575, Mar. 2009 .

[9] S. Inada et al., "Importance of gradients in membrane properties and electrical coupling in sinoatrial node pacing," PLoS ONE, vol. 9, no. 4, p. e94565, Apr. 2014.

Address for correspondence:

Stefano Severi

DEI, University of Bologna, Cesena, Via Venezia 52, 47521, Cesena (FC), Italy stefano.severi@unibo.it 